\title{
Detección molecular de patógenos entéricos en niños con diarrea en un hospital centinela de vigilancia de rotavirus en Chile
}

\section{Molecular detection of gastrointestinal pathogens among children under 5 years old with diarrhea in a hospital center for rotavirus sentinel surveillance in Chile}

\author{
Carolina Poulain ${ }^{1}$, Héctor Galeno², Sergio Loayza³, Natalia Vergara', Francisca Valdivieso4, Paulina Coria”, \\ Viviana Sotomayor ${ }^{1}$, María Elvira Simian 4 , Johanna Acevedo ${ }^{3}$ y Mauricio J. Farfán ${ }^{6}$
}

'Unidad de Vigilancia de Enfermedades Transmisibles, Departamento de Epidemiología, Ministerio de Salud de Chile.

${ }^{2}$ Laboratorio de Virus Entéricos, Instituto de Salud Pública.

${ }^{3}$ División de Planificación Sanitaria, Ministerio de Salud de Chile.

${ }^{4}$ Laboratorio de Microbiología Hospital Dr. Luis Calvo Mackenna.

5Unidad de Infectología, Hospital Dr. Luis Calvo Mackenna.

${ }^{6}$ Laboratorio de Biología Molecular, Hospital Dr. Luis Calvo Mackenna.

Conflicto de interés: los autores declaran no tener conflicto de interés.

Financiamiento: Departamento de Epidemiología, Ministerio de Salud de Chile y Hospital Dr. Luis Calvo Mackenna. Proyecto FONDECYT/ANID No 1200994 (MJF)

Recibido (segunda versión): 29 de diciembre de 2020 / Aceptado: 9 de enero de 2021

\section{Resumen}

Introducción: Las diarreas de causa infecciosa son un problema de salud pública, especialmente en niños bajo los cinco años. La identificación de los agentes etiológicos puede ser relevante para el manejo del cuadro clínico y, desde el punto de vista epidemiológico, para la implementación de medidas de control. Objetivo: Determinar la presencia de patógenos entéricos en niños bajo los cinco años que se hospitalizaron por diarrea aguda en uno de los centros centinelas de la red de vigilancia de rotavirus en Chile. Pacientes y Métodos: Estudio observacional en niños menores de cinco años que se internaron por cuadros de diarrea en el Hospital Dr. Luis Calvo Mackenna, durante diciembre del 2015 a diciembre del 2019, el que forma parte de la red de vigilancia de rotavirus del Ministerio de Salud de Chile. Las muestras fecales se analizaron mediante un test molecular, FilmArray GI ${ }^{\circledR}$ panel, que permite la detección de 22 patógenos entéricos virales, bacterianos y parasitarios. Resultados: Se analizaron 493 muestras fecales de niños con episodios de diarrea infecciosa, detectando al menos un patógeno en 427 muestras (87\%). De estas muestras positivas, se detectó solo un patógeno en 174 muestras (41\%) y dos o más patógenos en 253 muestras (59\%). En el grupo de niños bajo un año y el grupo entre uno y cuatro años hubo un predominio de infecciones causadas por virus gastroentéricos, siendo rotavirus y norovirus los virus más detectados en ambos grupos de edad. Las bacterias más frecuentes fueron EPEC (27\%), C. difficile (17\%), EAEC (14\%) y

\section{Abstract}

Background: Infectious diarrhea is still a major problem in public health, especially in children under 5 years of age. The identification of the etiologic agent is important for the clinical management of the diarrhea episode and, from the epidemiological point of view, to implement control measures. Aim: To determine the presence of gastrointestinal pathogens in children under five years of age with diarrhea in a Chilean rotavirus surveillance center. Methods: Observational study in children under five years of age who were hospitalized for diarrhea at the Dr. Luis Calvo Mackenna Hospital from December 2015 to December 2019. Molecular detection was performed using the FilmArray gastrointestinal (FilmArray $\mathrm{GI}^{\circledR}$ ) panel. Results: We analyzed 493 diarrheal stool samples of children, 427 samples (87\%) were positive and 66 samples (13\%) were negative. Of positive samples, 174 samples (41\%) and 253 samples $(59 \%)$ were positive for one or more pathogen, respectively. In children under one year and the group between one and four years there was a predominance of infections caused by enteric virus. Rotavirus and norovirus were the most common virus in both age groups. The most frequent bacteria were EPEC (27\%), C. difficile (17\%), EAEC (14\%) and Campylobacter (9\%). In parasites, Giardia lamblia and Cryptosporidium were identified, in 3\% and $1 \%$ of the total samples, respectively. Conclusions: The molecular detection system used allowed an increase in the detection of enteropathogens 
in children under five years of age. The information generated by this type of surveillance could help to characterize the episodes of diarrhea in the population and might be a tool to technically advise the authorities in the decision-making process for the implementation of control measures.

Keywords: molecular detection; enteric pathogens; diarrhea surveillance; children.

\section{Introducción}

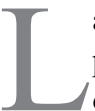

as infecciones gastrointestinales son un problema de salud pública mundial. En el año 2015, se estimó una incidencia de 688 millones de casos en el mundo con 499.000 muertes en niños bajo los 5 años. Si bien la implementación de medidas de higiene, prevención y tratamiento de la deshidratación han disminuido considerablemente la mortalidad de las diarreas infecciosas en países en desarrollo, la morbilidad y los costos asociados continúan siendo un problema de salud pública ${ }^{1}$.

La vigilancia epidemiológica y los estudios longitudinales permiten conocer y caracterizar los episodios de diarrea aguda en una población, además de entregar una base para la implementación de estrategias de control contra estas infecciones ${ }^{2}$. Uno de los grandes obstáculos para los estudios de vigilancia en diarrea es la elección de las herramientas diagnósticas para determinar su etiología. Si bien el cultivo es la técnica diagnóstica de referencia para la mayoría de los patógenos gastrointestinales, su bajo rendimiento y la dificultad para identificar algunos agentes (virus y parásitos, principalmente), han disminuido su uso, tanto en el diagnóstico rutinario como en estudios epidemiológicos ${ }^{3}$. El desarrollo de herramientas de biología molecular, no sólo ha permitido una mayor sensibilidad en la detección de patógenos, sino que sus plataformas de detección de múltiples agentes en una sola reacción ha facilitado la identificación de patógenos entéricos clásicos (Shigella, Salmonella y rotavirus), agentes de difícil cultivo (Campylobacter, Clostridium, virus y parásitos en general) y patógenos cuya identificación se basa en la detección de factores de patogenicidad específicos (Escherichia coli diarreogénicas) ${ }^{4,5}$. En este sentido, las técnicas de diagnóstico molecular múltiple han abierto un nuevo horizonte para conocer la etiología de las diarreas y comprender la epidemiología de los agentes responsables de estas infecciones ${ }^{6,7}$. Sin embargo, su uso como herramienta diagnóstica y epidemiológica ha generado una gran discusión en la interpretación de los resultados, considerando el aumento de patógenos detectados y la etiología de la diarrea ${ }^{8}$.

Considerando la escasa información epidemiológica que existe sobre las diarreas infecciosas en Chile, en este trabajo se utilizó una técnica de diagnóstico molecular para 22 patógenos gastrointestinales con el objetivo de determinar la etiología de la diarrea aguda en niños bajo los cinco años en uno de los centros centinelas que forman parte de la red de vigilancia de rotavirus de nuestro país.

\section{Método}

\section{Diseño global del estudio}

Se realizó un estudio retrospectivo, observacional, en niños bajo los cinco años que se internaron por diarrea aguda en el Hospital Dr. Luis Calvo Mackenna (HLCM) durante los meses de diciembre del año 2015 a diciembre del 2019. El HLCM, desde el año 2007 es un centro de vigilancia centinela de infecciones gastrointestinales por rotavirus del Ministerio de Salud de Chile (MINSAL), realizándose detección de rotavirus en los niños bajo cinco años consultantes por diarrea y que requieren hospitalización, al menos transitoria, para hidratación.

De forma rutinaria, las muestras se analizaron en HLCM mediante test rápido para rotavirus y un test de ELISA en el Laboratorio de Virología del Instituto de Salud Pública (ISP). A partir del año 2015, se incorporó el panel FilmArray $\mathrm{GI}^{\circledR}$ para el análisis de las muestras incluidas en esta vigilancia para ampliar el estudio etiológico de estas diarreas. Como fuente de datos se utilizaron los registros de esta vigilancia que incorporaba el resultado del panel FilmArray GI ${ }^{\circledR}$ y contaba con las siguientes variables: sexo, edad y resultados del laboratorio local y de referencia nacional.

\section{Definición de caso de diarrea}

Se definió como diarrea a tres o más evacuaciones líquidas o semilíquidas en las últimas $24 \mathrm{~h}$, de hasta 14 días de duración. Un caso de diarrea correspondió a un niño menor de cinco años ingresado a una sala de rehidratación, que recibió rehidratación oral o endovenosa o que estuvo en una sala de hospitalización por diarrea. Se excluyeron los niños cuya causa de hospitalización era distinta a la diarrea, una toma de muestra después de $48 \mathrm{~h}$ del ingreso o haber sido referido desde otro establecimiento donde permaneció más de $24 \mathrm{~h}$.

\section{Detección de enteropatógenos}

La toma de muestras se realizó al ingreso al HCLM y se procesó en el laboratorio de Biología Molecular de este recinto para la detección de 22 patógenos entéricos mediante el panel FilmArray GI ${ }^{\circledR}$ (BioFire, Inc., Salt Lake City, UT), que permite la detección de los siguientes patógenos: i) virus: (adenovirus F40/41, astrovirus, norovirus GI/ GII (NoV), rotavirus A (RV), sapovirus I, II, IV y V), ii) bacterias: Campylobacter spp., Clostridium difficile, Plesiomonas shigelloides, Salmonella spp., Yersinia enterocolitica, Vibrio parahaemolyticus, 
Vibrio vulnificus, Vibrio cholerae, Shigella spp., Escherichia coli enteroagregativa (EAEC), E. coli enteropatogénica (EPEC), E. coli enterotoxigénica (ETEC), E. coli productora de toxina Shiga (STEC)y E. coli enteroinvasora (EIEC), y iii) parásitos: Cryptosporidium spp, Cyclospora cayetanensis, Entamoeba histolytica y Giardia lamblia. Se definió como co-detección la presencia de más de un patógeno en una muestra.

\section{Análisis estadístico}

Se utilizó Stata 12 (Stata Corp, College Station, Texas) y Microsoft Excel 2013.

Aspectos éticos. Con respecto a la Ley $N^{\circ} 20.584$ que "Regula los derechos y deberes que tienen las personas en relación con acciones vinculadas a su atención en salud", este estudio no vulnera la mencionada ley, debido a que utiliza fuentes de datos secundarios del MINSAL, los cuales se recogen dando cumplimiento a su rol según las siguientes leyes previas: DFL N ${ }^{o} 1 / 2005$ del MINSAL y Ley $N^{\circ} 19.628$ sobre datos sensibles. El Comité de Ética Científico Pediátrico del Servicio de Salud Metropolitano Oriente aprobó la realización de este artículo el 26 de diciembre de 2019.

\section{Resultados}

Durante los cuatro años del periodo de estudio, un total de 611 casos cumplieron la definición de caso de diarrea para esta vigilancia. En 115 de ellos (19\%) no se obtuvo muestra fecal o la muestra fue insuficiente para el ensayo de laboratorio. Tres muestras resultaron invalidadas en

Tabla 1. Distribución por grupo etario de cepas bacterianas, parasitarias y virales detectadas en muestras fecales positivas de niños con diarrea ( $n=427$ ), analizados mediante el panel FilmArray $\mathrm{Gl}^{\circledR}$, Hospital Dr. Luis Calvo Mackenna, diciembre 2015-diciembre 2019

\begin{tabular}{|c|c|c|c|}
\hline \multirow[t]{2}{*}{ Patógeno } & \multicolumn{3}{|c|}{$\begin{array}{l}\text { Número de muestras positivas } \\
\text { (\% sobre el total de muestras) }\end{array}$} \\
\hline & $\begin{array}{c}<5 \\
(n=493)\end{array}$ & $\begin{array}{c}<1 \text { año } \\
(n=288)\end{array}$ & $\begin{array}{l}\text { 1-4 años } \\
(n=265)\end{array}$ \\
\hline Bacterias & $51(10 \%)$ & $22(8 \%)$ & $29(11 \%)$ \\
\hline Bacterias - Bacterias & $29(6 \%)$ & $7 \quad(2 \%)$ & $22(8 \%)$ \\
\hline Bacterias - Parásitos & $4 \quad(1 \%)$ & $0 \quad(0 \%)$ & $4(2 \%)$ \\
\hline Bacterias - Virus & $186(38 \%)$ & $81(28 \%)$ & 105 (40\%) \\
\hline Bacterias - Virus - Parásitos & 11 (2\%) & $0 \quad(0 \%)$ & 11 (4\%) \\
\hline Virus & $123(25 \%)$ & $50(20 \%)$ & $64(24 \%)$ \\
\hline Virus - Virus & $20 \quad(4 \%)$ & $8(3 \%)$ & 12 (5\%) \\
\hline Virus - Parásitos & $3 \quad(1 \%)$ & $0 \quad(0 \%)$ & $3(1 \%)$ \\
\hline Total de muestras & 427 & 177 & 250 \\
\hline
\end{tabular}

el panel FilmArray $\mathrm{GI}^{\circledR}$. En resumen, se analizaron 493 muestras correspondientes a igual número de casos.

La mediana de edad de los casos estudiados fue de 13 meses (rango de nueve días a cuatro años, diez meses), 228 (46\%) casos tenían menos de un año de edad, y 265 (54\%) entre uno y cinco años. El 55\% (270/493) de los casos eran hombres; y 45\% (223/493) mujeres.

En 427 muestras $(87 \%)$ se detectó al menos un enteropatógeno; siendo 66 muestras $(13 \%)$ negativas para los agentes virales, bacterianos y parasitarios analizados. De las 427 muestras positivas, en 174 de ellas (41\%) se detectó sólo un agente; 253 muestras positivas (59\%) presentaron co-detección de patógenos. En este último grupo. en 154 muestras (36\%) se detectaron dos patógenos; en 71 $(17 \%)$ tres patógenos; y en $28(6 \%)$ cuatro o más agentes. La detección virus-bacteria se observó en 186 muestras positivas (38\%); bacteria-bacteria en 29 muestras (6\%); virus-virus en 20 muestras (4\%); virus-bacteria-parásito en 11 muestras (2\%). La co-detección de bacteria-parásito y virus-parásito fue infrecuente (1\% en ambos grupos). En la Tabla 1 se resume la frecuencia de detección de cepas bacterianas, parasitarias y virales en los 427 casos positivos, según los grupos de edad analizados.

En la Figura 1 se presenta la frecuencia de detección de enteropatógenos en las muestras analizadas. De los $17 \mathrm{di}$ ferentes patógenos encontrados, todos se detectaron junto a algún otro agente. Los agentes con mayor porcentaje de co-detección, sobre el 90\%, fueron EPEC y EAEC, ambas con un $93 \%$. Por otra parte, los agentes que presentaron las más bajas frecuencias de co-detección fueron rotavirus (63\%), Salmonella (67\%) y EIEC (67\%).

Según el tipo de patógeno, los virus gastroentéricos se detectaron en $70 \%$ de las muestras (343/493); en $17 \%$ de los casos positivos a virus (59/343) se encontraron dos o más virus gastroentéricos. Los enteropatógenos bacterianos se detectaron en 57\% (281/493); en 32\% de estos casos positivos $(90 / 281)$ se detectaron dos o más patógenos bacterianos. Los protozoos parasitarios se detectaron en 4\% de las muestras analizadas (18/493) y sólo en un caso se detectaron dos protozoos simultáneos.

No se observaron diferencias significativas en la frecuencia de detección para virus, bacterias y parásitos entre ambos sexos.

Con respecto a la edad, no se encontraron diferencias significativas para virus y bacterias entre los dos grupos etarios establecidos. En el caso de los parásitos, si bien se encontró diferencias entre los dos grupos etarios, es importante mencionar que en el grupo de lactantes bajo un año no se detectaron parásitos.

Utilizando el panel FilmArray $\mathrm{GI}^{\circledR}$, se detectaron 409 virus, 386 bacterias y 19 parásitos. En la Tabla 2 se detallan los agentes detectados en las 427 muestras positivas para uno o más patógenos. De los agentes virales, rotavirus fue el virus más detectado y uno de los patógenos 


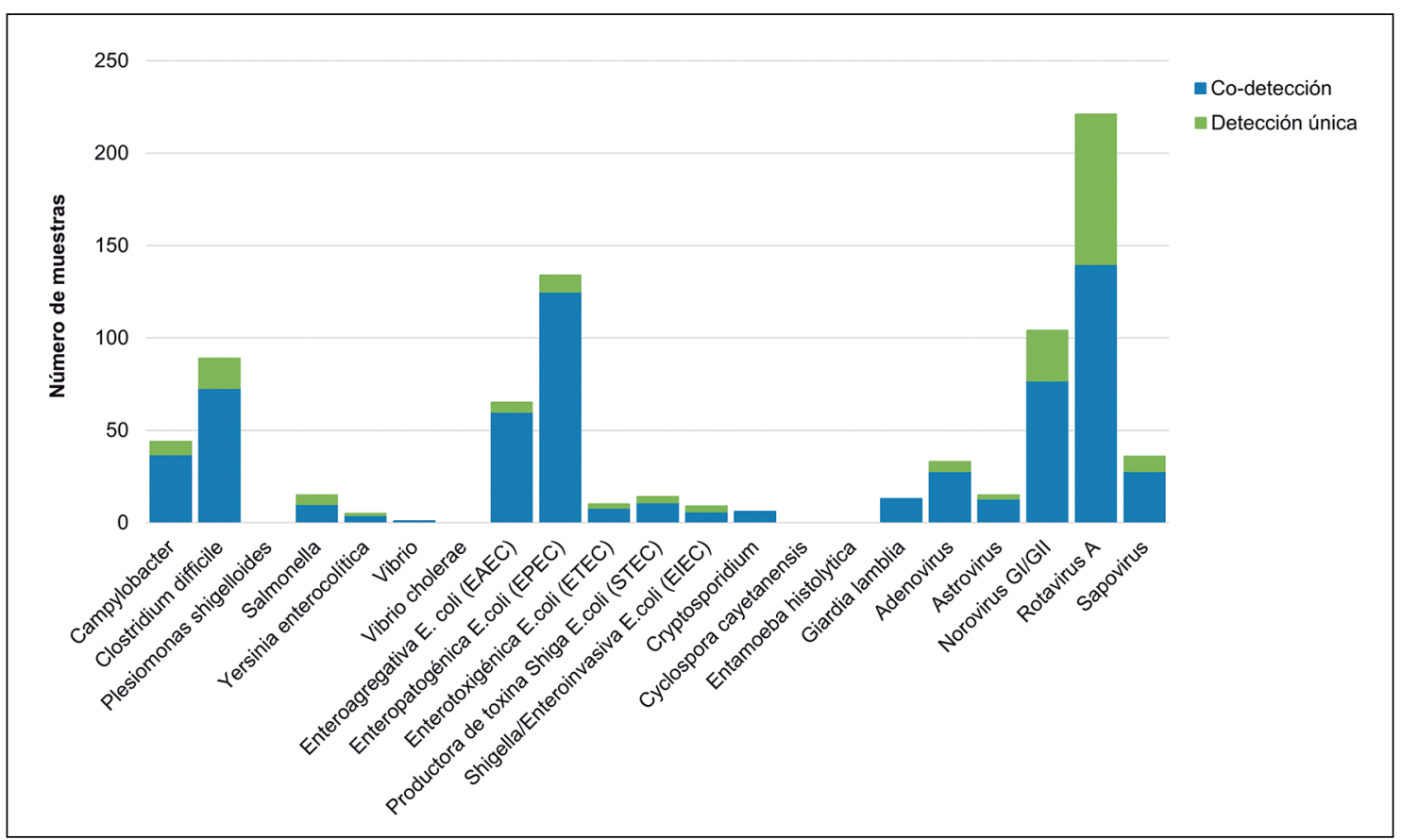

Figura 1. Frecuencia de detección de agentes enteropatógenos en niños con diarrea, desagregada por detección única y co-detección, Hospital Dr. Luis Calvo Mackenna, diciembre 2015 - diciembre 2019

Tabla 2. Número de detecciones y porcentaje sobre el total de muestras de niños con diarrea analizadas por el panel FilmArray GI ${ }^{\circledR}$, según patógeno entérico y grupo etario, Hospital Dr. Luis Calvo Mackenna, año diciembre 2015-diciembre 2019

\begin{tabular}{|c|c|c|c|}
\hline \multirow[t]{2}{*}{ Patógeno } & \multicolumn{3}{|c|}{ Número de detecciones positivas (\% sobre el total de muestras) } \\
\hline & $<5(n=493)$ & $<1$ año $(n=288)$ & $1-4$ años $(n=265)$ \\
\hline \multicolumn{4}{|l|}{ Bacterias } \\
\hline Campylobacter & $44(9 \%)$ & $7(2 \%)$ & $37(14 \%)$ \\
\hline Plesiomonas shigelloides & $0(0 \%)$ & $0(0 \%)$ & $0(0 \%)$ \\
\hline Salmonella spp. & $15(3 \%)$ & $5(2 \%)$ & $10(4 \%)$ \\
\hline Yersinia enterocolítica & $5(1 \%)$ & $3(1 \%)$ & $2(1 \%)$ \\
\hline E. coli enteroagregativa (EAEC) & $65(13 \%)$ & $22(8 \%)$ & $43(16 \%)$ \\
\hline E. coli enteropatogénica (EPEC) & $134(27 \%)$ & $46(16 \%)$ & $88(33 \%)$ \\
\hline E. coli enterotoxigénica (ETEC) & $10(2 \%)$ & $2(1 \%)$ & $8(3 \%)$ \\
\hline E. coli productora de toxina Shiga (STEC) & $14(3 \%)$ & $4(1 \%)$ & $10(4 \%)$ \\
\hline Shigella/E. coli enteroinvasiva (EIEC) & $9(2 \%)$ & $2(1 \%)$ & $7(3 \%)$ \\
\hline \multicolumn{4}{|l|}{ Parásitos } \\
\hline \multicolumn{4}{|l|}{ Virus } \\
\hline Adenovirus F40/41 & $33(7 \%)$ & $12(4 \%)$ & $21(8 \%)$ \\
\hline Astrovirus & $15(3 \%)$ & $7(2 \%)$ & $8(3 \%)$ \\
\hline Norovirus Gl/Gll & $104(21 \%)$ & $35(12 \%)$ & $69(26 \%)$ \\
\hline Rotavirus A & $221(45 \%)$ & $110(38 \%)$ & $111(42 \%)$ \\
\hline Sapovirus & $36(7 \%)$ & $9(3 \%)$ & $27(10 \%)$ \\
\hline Total de detecciones & 814 & 318 & 496 \\
\hline
\end{tabular}

*En cada celda está el número de muestras positivas para el respectivo agente (\% sobre el total de muestras analizadas). Debido a que se detectaron múltiples agentes en $60 \%$ de los casos, la sumatoria supera al total de casos. 
prevalentes en ambos grupos de edad, 38\% (110/288) en el grupo de lactantes bajo un año y $42 \%(111 / 265)$ en los niños de uno a cuatro años. En el grupo etario de uno a cuatro años se observó mayor frecuencia de norovirus (26\%; 69/265), comparado con los niños menores de un año $(12 \% ; 35 / 288)$.

De las 493 muestras incluidas en este trabajo, se analizaron 325 mediante el test inmunoquímico de ELISA. Al comparar el diagnóstico de rotavirus se observó que, en todas las muestras positivas para rotavirus por ELISA se detectó este virus por FilmArray $\mathrm{GI}^{\circledR}$, con excepción de dos muestras. Para el caso de las muestras negativas por ELISA, 10,5\% (21/199) resultaron ser positivas para rotavirus por el panel molecular.

Los agentes bacterianos más frecuentes fueron EPEC (27\%; 134/493), C. difficile (18\%; 89/493), EAEC (13\%; 65/493) y Campylobacter (9\%; 44/493). En los lactantes bajo un año, $C$. difficile se detectó en $19 \%(54 / 288)$ y EPEC en 16\% (46/288). En el grupo etario de uno a cuatro años de edad, se observó un aumento de EPEC
$(33 \% ; 88 / 265)$ y una disminución de $C$. difficile $(13 \%$; $35 / 265)$. Campylobacter spp fue detectado en $2 \%(7 / 288)$ de los lactantes bajo un año y en 14\% (37/265) de los mayores a un año.

Dentro de los parásitos, sólo se identificó Giardia lamblia y Cryptosporidium spp, en 3 y $1 \%$ del total de las muestras de niños bajo y sobre un año, respectivamente.

Según la estacionalidad, 35\% (137/386) de los agentes bacterianos se detectaron entre enero y marzo (meses de verano), principalmente las cepas de $E$. coli diarreogénicas y $C$. difficile. Por su parte, los casos con muestras positivas a Campylobacter spp, no presentaron un perfil estacional, detectándose en todos los meses del año. Con respecto a los agentes virales, se observó un aumento en la detección de norovirus en primavera y verano con respecto a otoño e invierno ( 80 versus 24 detecciones). En tanto que las detecciones de rotavirus aumentaron en otoño e invierno con respecto a primavera verano (126 versus 95 detecciones). En el caso de los parásitos, no fue posible establecer un patrón estacional (Figura 2).

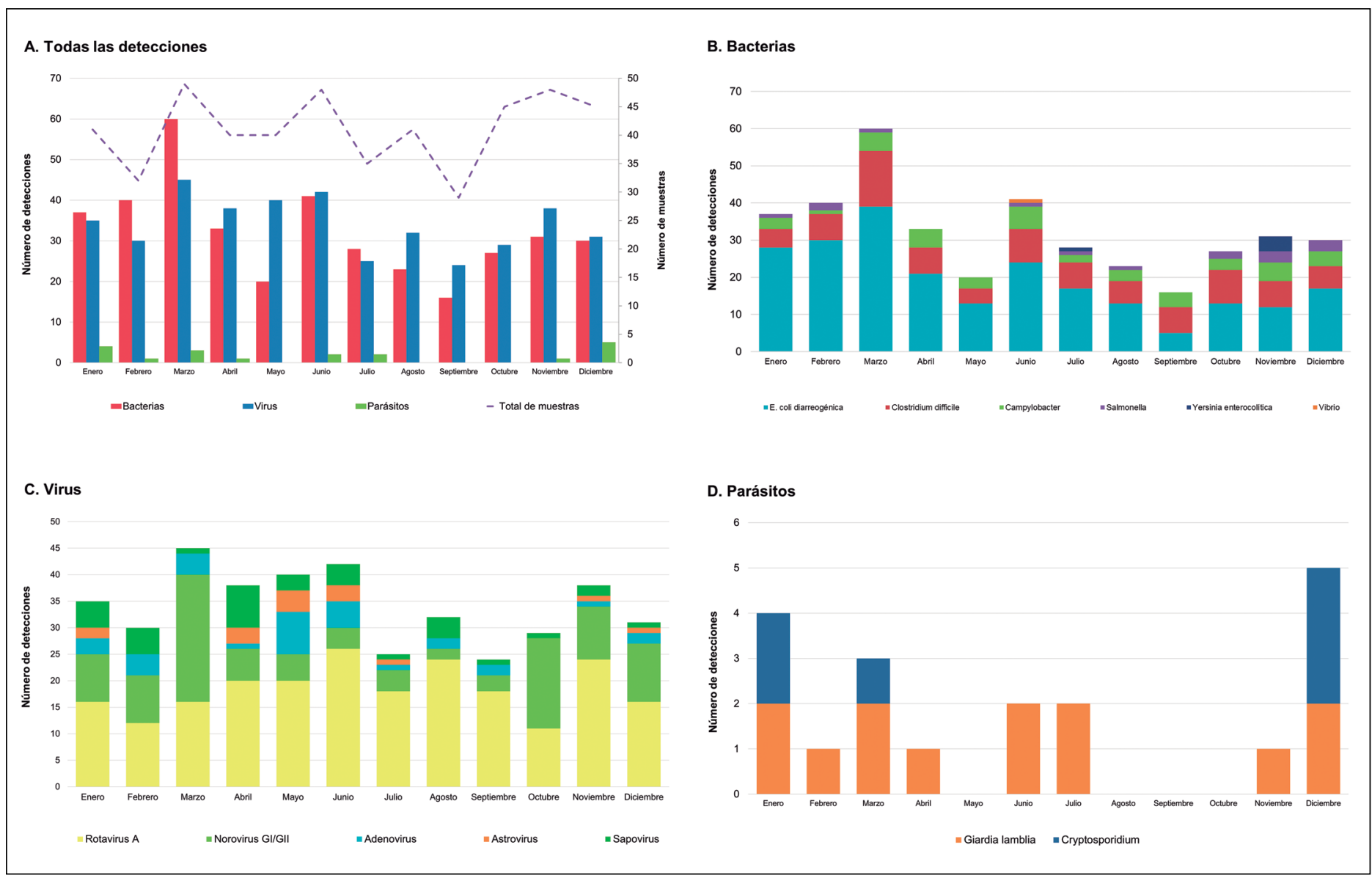

Figura 2. Número de detecciones por el panel FilmArray $\mathrm{Gl}^{\circledR}$, según mes de toma de muestra, Hospital Dr. Luis Calvo Mackenna, año diciembre 2015 -diciembre 2019. 
podría tener un estado inmunitario distinto a los casos estudiados en este estudio, por tener un mayor acceso a la vacuna. En ninguno de estos estudios, se contó con el estado vacunal de los casos.

Las especies bacterianas con mayor frecuencia fueron Campylobacter spp, C. difficile y las cepas de EAEC y EPEC. Sin embargo, estas bacterias se detectaron por sobre el $80 \%$ en co-infecciones, lo que dificulta la interpretación de su rol como agente etiológico de la diarrea. En el caso de las cepas de $E$. coli diarreogénicas, numerosos estudios han encontrado la presencia de algunos de sus patotipos, principalmente EAEC y EPEC, en niños sin diarrea ${ }^{2,16}$. Una situación similar se ha observado con $C$. difficile y Campylobacter spp, donde mediante el uso de técnicas de diagnóstico molecular múltiple se ha detectado la presencia de este microorganismo en niños bajo 5 años de edad sin síntomas de infección gastrointestinal ${ }^{7,18}$. En este sentido, es importante mencionar que la técnica utilizada en esta vigilancia es del tipo cualitativa, y dada su alta sensibilidad no permite discriminar entre enteropatógenos presentes de un episodio de diarrea anterior ó como parte de la microbiota intestinal de estos niños. El desarrollo de plataformas diagnósticas cuantitativas para enteropatógenos podría facilitar la interpretación de los resultados de co-infección y el desarrollo de algoritmos para apoyar el diagnóstico de las diarreas.

Nuestro estudio tiene limitaciones. Debido al diseño del mismo, no se realizó cultivo microbiológico de los enteropatógenos. Sin embargo, la plataforma diagnóstica utilizada, presenta valores de sensibilidad y especificidad altos en sus etapas de validación ${ }^{7}$. Por otra parte, el cultivo de la mayoría de los patógenos incluidos en el panel no se realiza de forma convencional. Sin embargo, la escasez de estudios con técnicas moleculares múltiples, que determinen la prevalencia de estos agentes en niños sanos, dificulta la atribución de causalidad a la detección de algunos de estos microorganismos en los niños con diarrea.

La vigilancia epidemiológica con técnicas moleculares mejora el rendimiento etiológico de las diarreas en niños bajo los cinco años, permitiendo la identificación de una gran variedad de patógenos intestinales. Al implementar esta técnica en todos los establecimientos centinela de rotavirus, se podría responder en forma oportuna a eventos inusitados como brotes, lo que permitiría mantener la vigilancia de rotavirus si se implementa la vacuna y además responder a cambios epidemiológicos hoy no vislumbrados, que pudieran requerir a futuro de modificaciones de las acciones de salud pública. 


\section{Referencias bibliográficas}

1.- Mokomane M, Kasvosve I, De Melo E, Pernica J G. The global problem of childhood diarrhoeal diseases: emerging strategies in prevention and management. Ther Adv Infect Dis. 2018; 5 (1): 29-43. doi: $10.1177 / 2049936117744429$.

2.- Kotloff K L. The burden and etiology of diarrheal illness in developing countries. Pediatr Clin North Am. 2017; 64 (4): 799-814. doi: 10.1016/j.pcl.2017.03.006.

3.- Chi C Y, Liao L N, Ho C M, Chou C H, Ho M W, Wang J H. Epidemiology, clinical features, and microbiology of patients with diarrhea in community clinics in Taiwan. J Microbiol Immunol Infect 2018; 51 (4): 527-34 doi: 10.1016/j.jmii.2017.05.003.

4.- Konaté A, Dembélé R, Kagambèga A, Soulama I, Kaboré W A D, Sampo E, et al. Molecular characterization of diarrheagenic Escherichia coli in children less than 5 years of age with diarrhea in Ouagadougou, Burkina Faso. Eur J Microbiol Immunol (Bp). 2017; 7 (3): 220-8. doi: 10.1556/1886.2017.00011.

5.- Sánchez G J, Mayta H, Pajuelo M J, Neira K, Xiaofang L, Cabrera L, et al. Epidemiology of sapovirus infections in a birth cohort in Peru for the Sapovirus Working Running title: Sapovirus infections in a birth cohort. Clin Infect Dis. 2018; 66 (12): 1858-63 doi: 10.1093/cid/ cix1103.

6.- Stockmann C, Pavia A T, Graham B, Vaughn M, Crisp R, Poritz M A, et al. Detection of 23 gastrointestinal pathogens among children who present with diarrhea. J Pediatric Infect Dis Soc. 2017; 6 (3): 231-8. doi: 10.1093/jpids/piw020.

7.- Buss S, Leber A, Chapin K, Fey P, Bankowski $\mathrm{M}$, Jones M, et al. Multicenter evaluation of the BioFire FilmArray gastrointestinal panel for etiologic diagnosis of infectious gastroenteritis. J Clin Microbiol. 2015; 53 (3): 915-25. doi: 10.1128/JCM.02674-14.

8.- Kotloff K L, Platts-Mills J A, Nasrin D, Roose A, Blackwelder W C, Levine M M. Global burden of diarrheal diseases among children in developing countries: Incidence, etiology, and insights from new molecular diagnostic techniques. Vaccine. 2017; 35 (49 Pt A): 6783-9. https://doi.org/10.1016/j. vaccine.2017.07.036.

9.- Lucero Y, Mamani N, Cortés H, Peña A, Vergara R, O'Ryan M. Rotavirus genotypes in children with gastroenteritis assisted in two public hospitals from Chile: viral strains circulating in a country without a universal vaccination against rotavirus. Rev Chil infectología 2012; 29 (2): 142-8. http://dx.doi. org/10.4067/S0716-10182012000200004.

10.- Ministerio de Salud de Chile. Informe de Rotavirus. Chile, año 2015. Santiago; 2015. Disponible en: http://epi.minsal.cl/wp- content/ uploads/2016/11/2016.11.18_ROTAVIRUSINFORME-ANUAL.pdf (fecha de acceso: 9 de abril de 2018).

11.- Sindhu K, Babji S, Ganesan S. Impact of rotavirus vaccines in low and middle-income countries. Curr Opin Infect Dis. 2017; 30 (5): 473-81. doi: 10.1097/QCO.0000000000000397.

12.- Programa Nacional de Inmunizaciones. Recomendaciones para la vacunación de pacientes con necesidades especiales por patologías o situaciones de riesgo. Chile, año
2018.Santiago; 2018. Disponible en: https:// vacunas.minsal.cl/wp-content/uploads/2019/02/ MANUAL-VACUNAS-ESPECIALES.pdf.

13.- O'Ryan M. Rotavirus vaccines: a story of success with challenges ahead. F1000Research. 2017; 6 (0): 1517. doi: 10.12688/ f1000research.11912.1.

14.- Muñoz A, Abarca K, Luchsinger V, Valenzuela M, Jiménez J. Declaración del Comité Consultivo de Inmunizaciones (CCI) de la Sociedad Chilena de Infectología respecto a vacuna anti-rotavirus: Marzo 2006. Rev Chil Infectología 2006; 23 (2): 124-7. http://dx.doi. org/10.4067/S0716-10182006000200004.

15.- Ali Z, Harastani H, Hammadi M, Reslan L, Ghanem S, Hajar F, et al. Rotavirus genotypes and vaccine effectiveness from a sentinel, hospital-based, surveillance study for three consecutive rotavirus seasons in Lebanon. PLoS One. 2016; 11 (8):1-18. doi: 10.1371/ journal.pone. 0161345 .

16.- Farfán M, Piemonte P, Henríquez J, Candia E, Torres J. Panel Filmarray $\mathrm{GI}^{\circledR}$ en la detección de patógenos entéricos en deposiciones: experiencia preliminar. Rev Chilena Infectol. 2016; 89-91http://dx.doi.org/10.4067/S071610182016000100016.

17.- Pelkonen T, Dos Santos M, Roine I, Dos Anjos E, Freitas C, Peltola H, et al. Potential diarrheal pathogens common also in healthy children in Angola. Pediatr Infect Dis J. 2017; 1. doi: 10.1097/INF.0000000000001781.

18.- Nicholson M, Van Horn G, Tang Y, Vinjé J, Payne D, Edwards K. Using multiplex molecular testing to determine the etiology of acute gastroenteritis in children. J Pediatr. 2016; 176 (2): 50-6. doi: 10.1016/j.jpeds.2016.05.068. 